\title{
Criatividade e Grafos Existenciais em C. S. Peirce
}

[Creativity and Existential Graphs in C. S. Peirce]

\author{
José Renato Salatiel『
}

Resumo: O raciocínio lógico dedutivo é comumente definido como analítico e explicativo, no sentido em que nada acrescenta, em sua conclusão, além daquilo já contido nas premissas do argumento. Contudo, duas importantes conclusões da filosofia da lógica de Charles S. Peirce contestam essa suposta trivialidade do raciocínio dedutivo: (i) dedução é matéria de experimento e observação; e (ii) dedução é um tipo de raciocínio diagramático. O objetivo deste trabalho é mostrar como essas duas conclusões estão interligadas e como, juntas, podem elucidar aspectos criativos da lógica. Para isso, serão discutidas as seguintes teses. Primeiro, a prova dedutiva inclui uma etapa criativa (Peirce a chama de dedução teoremática) que explica como um raciocínio analítico pode, em alguns casos, ser surpreendente em suas conclusões. Segundo, sistemas formais que incluem representações diagramáticas, como os Grafos Existenciais, são mais eficientes em representar inferências dedutivas do tipo teoremáticas.

Palavras-chave: lógica diagramática; dedução; criatividade; Charles S. Peirce; grafos existenciais.

Abstract: Deductive reasoning is usually understood as analytical and explanatory, in the sense that it adds nothing to the conclusion that is not already contained in the premises of the argument. However, two important conclusions of Charles S. Peirce's philosophy of logic challenge the supposed triviality of deductive reasoning: (i) deduction is a matter of experiment and observation; and (ii) deduction is a kind of diagrammatic reasoning. The aim of this paper is to show how these two conclusions are interconnected and how together they can elucidate some creative aspects of logic. To this end, the following theses will be discussed. First, deductive proof includes a creative step (Peirce calls it theorematic deduction) which explains how analytical reasoning can be, in some cases, surprising in its conclusions. Second, formal systems that include diagrammatic representations, such as Existential Graphs, are more efficient to represent deductive inferences of the theorematic type.

Keywords: diagrammatical logic; deduction; creativity; Charles S. Peirce; existential graphs

${ }^{*}$ Professor Adjunto da Universidade Federal do Espírito Santo (UFES). Doutor em Filosofia pela Pontifícia Universidade Católica de São Paulo (PUC-SP). E-mail: jrsalatiel@hotmail.com. ORCID: https://orcid.org/0000-00015858-1248. 


\section{Introdução}

O raciocínio lógico é comumente dividido em dedutivo e indutivo, onde somente a dedução garante a certeza da conclusão que se segue de premissas verdadeiras. Esta é sua vantagem e a força epistêmica das disciplinas formais, a lógica e a matemática. Se uma prova é feita de maneira correta, mediante a aplicação de um conjunto de axiomas e regras de inferência, a verdade da conclusão será uma consequência lógica de premissas verdadeiras.

Isso quer dizer que o raciocínio dedutivo nada acrescenta, em sua conclusão, além daquilo já veiculado nas premissas do argumento. A dedução, neste sentido, é puramente analítica e tautológica, ao contrário da indução, que faz avançar a inquirição científica acrescentando informações ao término do processo inferencial.

Para evitar problemas do uso de critérios não puramente lógicos de definição, a diferença, conforme estabelecida por Frege no Begriffsschrift, é explicada em termos do método de prova adotado em cada raciocínio. 11 Visto por seu aspecto formal, define-se dedução a partir da noção de consequência lógica: um argumento é dedutivamente válido se não é o caso de, sendo suas premissas, verdadeiras, sua conclusão ser falsa. Sistemas lógicos formais são assim construídos tendo como objetivo a demonstração da validade sintática e semântica de classes de argumentos dedutivos. Provas dedutivas são então obtidas pela aplicação mecânica de axiomas e regras de inferência, ficando a cargo de investigações metalógicas o estudo das propriedades formais desses sistemas, como correção e completude.

Mas essa ênfase no método de prova em detrimento do processo dedutivo deixa de explicar como uma conclusão dedutiva pode ser não trivial, mas trazer elementos novos à inquirição, impor dificuldades e produzir descobertas. Em síntese, como um procedimento puramente analítico pode ser surpreendente? Isso é o que Hintikka (1973) chamou de o "escândalo da dedução" e Shin $(2015,2016)$, de o "mistério da dedução".

Neste artigo chamamos a atenção para aspectos criativos do processo dedutivo que, em grande parte, têm sido negligenciados pela lógica moderna. O objetivo será discutir as seguintes teses a esse respeito: (i) a tese de que esse elemento de criatividade do raciocínio dedutivo pode ser explicado pelo conceito de dedução

\footnotetext{
${ }^{1}$ FREGE, 1967. p. 5-8.
} 
teoremática de Charles S. Peirce; e (ii) que o sistema lógico diagramático desenvolvido pelo filósofo, chamado Grafos Existenciais, seria mais eficiente para representálo e evidenciá-lo, em um método de prova ao estilo da dedução natural.

Para isso, o artigo divide-se em três seções. Na primeira examinamos a divisão triádica que Peirce faz do raciocínio lógico, distinguindo dedução, indução e abdução, no contexto daquilo que chamaremos de sua "fase silogística". $\mathrm{Na}$ segunda seção, discutimos duas importantes consequência dos estudos de lógica de Peirce em sua "fase diagramática": (i) dedução é matéria de experimento e observação; e (ii) dedução é um tipo de raciocínio diagramático. E analisamos a tese de que o raciocínio teoremático é um elemento criativo ou abdutivo na prova dedutiva. Finalmente, na terceira seção, mostramos como um sistema lógico diagramático, como os Grafos Existenciais, é mais eficiente para o exame dessa etapa criativa na prova matemática.

\section{1 - Três tipos de raciocínio: fase silogística}

Charles S. Peirce é hoje reconhecido como um dos mais importantes nomes da lógica moderna. Entretanto, diferentemente da tradição logicista de Frege, Hilbert e Russell, para ele interessava à lógica, mais do que a invenção de sistemas axiomáticos de cálculo, a avaliação de métodos das ciências. Essa característica explicaria a ênfase, em sua obra, na investigação de processos inferenciais e no desenvolvimento de sistemas lógicos diagramáticos, como os Grafos Existenciais.

Desde seus primeiros trabalhos em lógica, em 1865, até a fase madura, em 1911, Peirce defendeu uma divisão de três - e apenas três - tipos de raciocínio lógico: dedução, indução e abdução (também chamada de retrodução ou hipótese) $)^{2}$. A maneira como definiu essas formas lógicas argumentativas, contudo, evoluiu ao longo de sua carreira, concluindo, ao final, que essas distinções seriam menos estáticas do que aparentam. A própria dedução, ao incorporar traços hipotéticos-criativos e experimentais, como veremos adiante, teria elementos característi-

\footnotetext{
${ }^{2}$ Essential Peirce. HOUSER, Nathan; KLOESEL, Christian (eds.). Vol. 1. Bloomington: Indiana University Press, 1992, p. 333. Doravante, citado como EP 1 seguido da página.

${ }^{3}$ Para uma análise dessa evolução, cf. RODRIGUES, 2011, e PIETARINEN \& BELLUCCI, 2014.
} 
cos, respectivamente, dos raciocínios abdutivos e indutivos. 3

Para os propósitos deste trabalho, o conceito de dedução em Peirce será examinado em duas fases distintas, que chamaremos de silogística e diagramática. Esta última, mais importante para os objetivos desta pesquisa, é analisada em detalhes na próxima seção.

Por fase silogística compreendemos, aproximadamente, o período de 1868 a 1885 . Nesse período, Peirce estabelece sua divisão triádica dos argumentos com base em formas silogísticas, na qual o conceito de dedução não difere das definições mais tradicionais.

Exemplos mais bem constituídos dessa primeira fase são encontrados nos artigos que compõem as chamadas Ilustrações da Lógica da Ciência, publicadas entre 1877 e 1878 na revista Popular Science Montly. Neles Peirce define duas classes de argumentos ou inferências lógicas e as apresenta em uma terminologia kantiana: analíticos/explicativos (dedução) e sin- téticos/ampliativos (indução e hipótese).

Os argumentos da segunda classe, sintéticos, são derivados da primeira (argumentos analíticos) por um método de transposição de premissas de um silogismo na forma típica AAA-1 (Barbara). Para isso são feitas mudanças nas quais duas proposições quantificadas do silogismo ("Todo S é P") são tornadas singulares ("Este $\mathrm{S}$ é $\mathrm{P}$ "), e o termo menor, "S", passa a ser analisado como uma amostra do termo maior, "P", uma população. Uma segunda forma de raciocínio lógico, a indução, é então obtida da primeira, dedução, transpondo a premissa maior (regra) para a conclusão, e a conclusão (resultado) para a premissa menor (o "caso" permanecendo nas premissas). O método é em seguida repetido para derivar a terceira forma de raciocínio, a hipótese, obtendo-se assim, duas diferentes formas não-dedutivas. Esses três tipos de raciocínio são apresentados no quadro a seguir:

\begin{tabular}{|l|l|l|}
\hline \multicolumn{1}{|c|}{ DEDUÇÃO } & \multicolumn{1}{|c|}{ INDUÇÃO } & \multicolumn{1}{c|}{ HIPÓTESE } \\
\hline $\begin{array}{l}\text { Regra: Todos os feijões } \\
\text { desta sacola são brancos. }\end{array}$ & $\begin{array}{l}\text { Caso: Estes feijões são } \\
\text { desta sacola. }\end{array}$ & $\begin{array}{l}\text { Regra: Todos os feijões } \\
\text { desta sacola são brancos. }\end{array}$ \\
\hline $\begin{array}{l}\text { Caso: Estes feijões são } \\
\text { desta sacola. }\end{array}$ & $\begin{array}{l}\text { Resultado: Estes feijões } \\
\text { são brancos. }\end{array}$ & $\begin{array}{l}\text { Caso: Estes feijões são } \\
\text { brancos. }\end{array}$ \\
\hline $\begin{array}{l}\text { Resultado: Estes feijões } \\
\text { são brancos. }\end{array}$ & $\begin{array}{l}\text { Regra: Todos os feijões } \\
\text { desta sacola são brancos. }\end{array}$ & $\begin{array}{l}\text { Resultado: Estes feijões } \\
\text { são desta sacola. }\end{array}$ \\
\hline
\end{tabular}

Quadro 1: Tipos de raciocínio em formas silogísticas (EP 1, 188). 
Como resultado, dedução, diz Peirce, consiste em mera "aplicação das regras gerais [premissas] a casos particulares [conclusão]" (EP 1, 187), enquanto a indução generaliza a partir de uma amostra e a hipótese supõe uma regra geral. Uma dedução válida, segundo o lógico, é aquela na qual, sendo as premissas verdadeiras, a conclusão seguirá, necessariamente, a verdade das premissas. Demonstrações matemáticas usam esse tipo de raciocínio. Já inferências sintéticas, empregadas nas ciências naturais, não são necessárias e diferem da dedução por serem ampliativas, ou seja, são "as únicas inferências que aumentam nosso conhecimento real" (EP 1, 161162).

Nesse primeiro estágio, não obstante a definição de dedução e indução não diferirem das concepções mais tradicionais, a descoberta da hipótese como uma inferência distinta das demais é um considerável avanço na lógica moderna. Mais tarde, chamando-a de abdução, Peirce dirá que esse tipo de inferência se caracteriza por ser a única que acrescenta elementos novos e criativos à inquirição. Será, portanto, o único tipo de inferência verdadeiramente sintética, à parte da indução, e não poderia, como tal, ser plenamente capturada na forma lógica silogística (CP 2.102) $)^{4}$ ou sequer inteiramente em uma forma lógica, por envolver traços irracionais, de instinto e insight (CP 5.181 e 5.188).

Essa divisão dos tipos de raciocínio teria ainda um papel maior na economia da inquirição científica, na medida em que Peirce compreendia serem, mais do que formas lógicas distintas, estágios do método científico. Isso se deve ao fato de ele considerar, em seu sistema filosófico, a lógica como uma ciência normativa, isto é, um processo de inquirição regido por normas que regulam a conduta de seus agentes em direção a um fim (a verdade). Neste contexto, cada tipo de raciocínio corresponderia a um estágio de investigação: a abdução fazendo conjecturas, a dedução extraindo consequências lógicas testáveis - prováveis ou necessárias - dessas hipóteses e a indução, realizando testes experimentais de conjuntos de hipóteses selecionadas. Dessa maneira, o processo criativo iniciado com a abdução, no âmbito da inquirição científica, é continuado pelos demais estágios - dedução e indu-

\footnotetext{
${ }^{4}$ PEIRCE, Charles Sanders. Collected Papers. 8 vols. HARTSHORNE, Charles; HEISS, Paul and BURKS, Arthur (eds.). Cambridge: Harvard University Press, 1931-1958. Citado como CP, seguido do número do volume e do número do parágrafo.
} 
ção.

Tanto esse caráter metodológico da abdução quanto sua função criativa, mencionada anteriormente, são desenvolvidas por Peirce em escritos posteriores, no qual a abdução "[...] é o único processo pelo qual um novo elemento pode ser introduzido no pensamento" (HPL, p. 239 $9^{5}$, cf. EP 2, p. 216 6.7

A concepção peirciana de dedução em termos silogísticos, contudo, permaneceria quase inalterada até 1885, com a publicação de "On the algebra of logic: a contribution to the philosophy of notation". 8 Nesse período, que chamaremos de fase diagramática, os resultados de seus estudos em lógica algébrica e semiótica o levariam a conceber a lógica dedutiva como uma atividade de experimento com diagramas.

\section{2 - Dois tipos de dedução: fase diagramática}

O principal avanço no conceito de dedução viria com o desenvolvimento da lógica algébrica. Entre 1870 a 1885 Peirce estendeu a lógica booleana para uma álgebra de relações, próxima daquilo que hoje entendemos como lógica da primeira ordem. Outro traço importante dessa mudança se deve à elaboração de sua doutrina semiótica, que expande as representações para além do escopo discursivo e simbólico.

Peirce concebia sistemas de representação multiformes, semióticos, que incluíam não somente signos simbólicos, mas signos icônicos..$^{9}$ A diferença é que, enquanto símbolos demandam uma convenção ou regra geral para serem interpretados, como as letras e palavras do alfabeto latino, os ícones estabelecem uma relação de similaridade qualitativa com seu objeto, como por exemplo, a silhueta do logotipo marca Apple que parece uma maçã mordida.

\footnotetext{
${ }^{5}$ PEIRCE, Charles Sanders. Pragmatism as a principle and method of right thinking: the 1903 Harvard lectures on pragmatism. TURRISI, Patricia Ann (ed.). New York: State University of New York Press, 1997. Citado como HLP, seguido do número da página.

${ }^{6}$ PEIRCE, Charles Sanders. Essential Peirce. The PEIRCE Edition Project (eds.). Vol. 2 (1893-1913). Bloomington: Indiana University Press, 1998. Citado como EP 2, seguido do número da página.

${ }^{7}$ Para uma análise completa desse quadro, cf. ANDERSON, 1987, cap. 2.

${ }^{8}$ Artigo publicado originalmente em American Journal of Mathematics, 7: 180-202 (CP 3.359-403).

${ }^{9}$ Nesta divisão mais conhecida, dos signos em relação aos seus objetos, há ainda o índice, que denota seu objeto por uma relação de contiguidade. Para uma visão mais completa da semiótica peirciana, cf. LISZKA, 1996.
} 


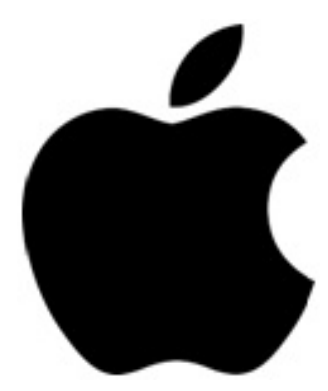

Imagem 1: Logotipo da Apple.

Fonte: <https://pt.wikipedia.org/wiki/Ficheiro:Apple_logo_black.svg>

Para Peirce, “[...] em um perfeito sistema de notação lógica, signos destes vários tipos devem ser todos empregados" (EP 2, 227). Mas, para ele, um tipo específico de signo icônico, o diagrama, é essencial ao raciocínio dedutivo. A diferença é que, enquanto uma imagem representa aspectos qualitativos de seu objeto, como forma e cor, um diagrama representa estruturas e relações formais (CP 2.279). E, assim como uma imagem, esses aspectos formais podem ser diretamente e imediatamente observados através da percepção visual.

De modo geral, diagrama são objetos concretos, visuais, por meio dos quais observamos relações estruturais e abstratas do raciocínio dedutivo. Os exemplos mais comuns, e de uso mais abrangente em matemática, são os diagramas usados na geometria euclidiana. Por exemplo, a prova para a proposição I.16 ("Se, dado um triângulo qualquer, tendo sido prolongado um de seus lados, então o ângulo exterior é maior do que cada um dos ângulos interiores e opostos"). A prova dessa proposição é um signo icônico que exibe, visualmente, no diagrama particular, relações espaciais entre os elementos das premissas e da conclusão.

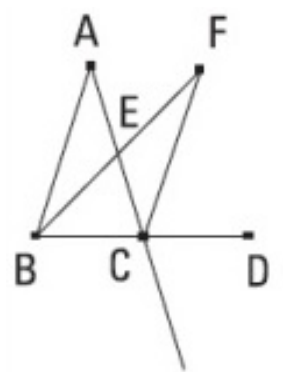

G 
O desenho do triângulo é um token que possibilita a observação, experimentação e manipulação de relações puramente lógicas. Para Peirce, não é um mero auxílio à imaginação, mas um elemento essencial à demonstração de um teorema matemático.

Em lógica, os diagramas mais conhecidos são os de Euler-Venn, usados frequentemente em manuais de lógica. Eles permitem visualizar diretamente a conclu- são de um argumento a partir de suas premissas, como no seguinte exemplo de um silogismo Celarent. Observando a primeira premissa, na qual o conjunto $M$ está excluído do conjunto P ("Nenhum M é P"), e a segunda premissa, na qual o conjunto $S$ está contido no conjunto M ("Todo S é M"), visualizamos diretamente a conclusão de que todo o conjunto S está, portanto, excluído do conjunto $\mathrm{P}$ (“Nenhum S é P"):

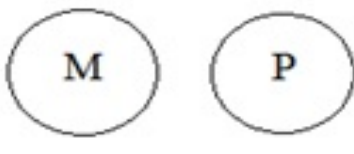

Premissa (1) Nenhum $M$ é $P$
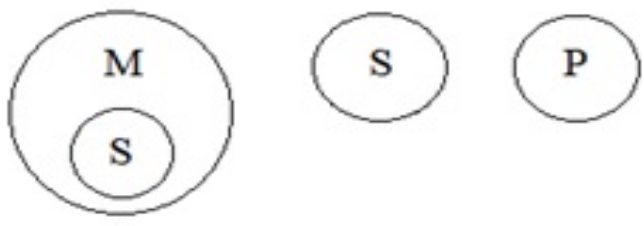

Conclusão

Nenhum S é P

Todo S éP

Imagem 3: Diagrama de Euler para o silogismo Celarent.

Contudo, em Peirce a noção de diagramas é abrangente o bastante para incluir formalizações simples, como a do silogismo acima representado em sua forma discursiva, no qual as letras assumem um caráter icônico, ou, como diz, Peirce, nesses diagramas "algébricos", as letras assumem função icônica. ${ }^{10}$ As letras, neste caso, operam como diagra- mas que representam as relações lógicas entre os termos menor (S), maior (P) e médio $(\mathrm{M})$ no silogismo:

\section{Nenhum $M$ é $P$ \\ Todo $S$ é $P$ \\ Nenhum $S$ é $P$}

A diferença é que, enquanto em um sistema notacional fortemente

\footnotetext{
${ }^{10}$ CP 4.530; HLP, 30, PM, 46-47 (PEIRCE, Charles S. Philosophy of Mathematics: selected writings. MOORE, Matthew E. (ed.). Indiana University Press: Bloomington and Indianapolis, 2010. Citado como PM, seguido do número do parágrafo.
} 
icônico, como nos diagramas de Euler-Venn, as relações entre as partes do argumento são percebidas diretamente, no caso dos sistemas discursivos a prova depende do entendimento de regras ou convenções, que são características de signos simbólicos.

É essa generalização do conceito de diagrama que o leva a resolver o problema das configurações não-triviais de uma dedução, conforme diz na seguinte passagem:

Há muito tempo tem sido um enigma como, por um lado, a matemática pode ser puramente dedutiva em sua natureza e obter suas conclusões apoditicamente, enquanto que, por outro lado, apresenta uma série fértil e aparentemente interminável de descobertas surpreendentes, tal como qualquer ciência observacional. Tem havido várias tentativas de resolver esse paradoxo explicando uma ou outra destas asserções, mas sem sucesso. A verdade, contudo, parece ser a de que todo raciocínio dedutivo, mesmo um simples silogismo, envolve um ele- mento de observação; isto é, dedução consiste na construção de um ícone ou diagrama das relações, cujas partes devem apresentar uma completa analogia com aquelas partes do objeto de raciocínio, de experimentar sobre uma imagem na imaginação e de observar o resultado, de forma a descobrir relações despercebidas e ocultas entre as partes. (EP 2, 227; cf. NEM IV, $10^{11}$ ).

Para Peirce, todo pensamento dedutivo (matemático) é um pensamento diagramático, isto é, envolve o emprego de diagramas. Diagrama, por sua vez, deve ser entendido de um modo geral o suficiente para abranger até mesmo uma notação simbólica, na qual os signos adquirem funcionalidade icônica no processo, conforme dito anteriormente.

Outra consequência, de caráter epistemológico, é que o raciocínio dedutivo envolve também observação e experimento, ainda que seja de um objeto abstrato e imaginado como um diagrama. Tal conclusão contraria uma certa compreensão da lógica matemática que a distingue das ciências

\footnotetext{
${ }^{11}$ PEIRCE, Charles S. The new elements of mathematics by Charles S. Peirce. 4 vols. EISELE, Carolyn (ed.). Bloomington: Indiana University Press, 1976. Citado como NEM seguido do volume e número da página.
} 
empíricas por empregar procedimentos puramente analíticos, mas que bem poderia ser aceita por aqueles que rejeitam uma clara distinção entre analítico e sintético.

Segundo Peirce, a lógica dos relativos (das relações em Russell) havia mostrado que a lógica formal envolve uma atividade de experimentação e manipulação de diagramas. Esses diagramas podem ser objetos físicos, mas, como diz Peirce, o experimento consiste em "[...] experimentar sobre uma imagem na imaginação e de observar o resultado, de forma a descobrir relações despercebidas e ocultas entre as partes" (Ibidem). Esse é um procedimento criativo, feito sobre a imaginação a partir da observação (ou não) de um token.

Mas nem toda dedução envolve, obviamente, um procedimento criativo. Em alguns casos a conclusão é facilmente derivada, como no argumento Celarent, do exemplo acima, representado em diagramas de Euler. Esse tipo de dedução Peirce chama de corolarial, que é aquela na qual as informações da conclusão são apenas extraídas das premissas. Para resolver o "escândalo" ou "mistério" de como a dedução pode ser cria- tiva, porém, é preciso observar um tipo de dedução que Peirce chama de teoremática. ${ }^{12}$ A diferença é explicada da seguinte forma:

Dedução corolarial é onde é somente necessário imaginar qualquer caso em que as premissas são verdadeiras para perceber imediatamente que a conclusão é válida naquele caso [...]. Dedução teoremática é a dedução em que é necessário fazer um experimento na imaginação sobre a imagem das premissas para, como resultado desse experimento, fazer deduções corolariais da verdade da conclusão (NEM IV, 38; cf. CP 2.267, CP 5.162 e CP 4.572).

O "experimento na imaginação" feito sobre "a imagem das premissas" - Peirce fala também de um experimento engenhoso (ingenious experiment) sobre o diagrama (CP 2. 267) e da introdução de uma "ideia externa" (foreign idea) (NEM IV, 42) - é o que distingue uma dedução teoremática de uma puramente corolarial.

\footnotetext{
${ }^{12}$ Peirce considerava ser sua primeira maior descoberta sobre o método matemático (NEM, IV, 49), cuja originalidade foi amplamente reconhecida e debatida por comentadores, entre eles HINTIKKA, 1980, KETNER, 1985 e LEVY, 1997.
} 
Após esse passo teoremático, uma prova segue conforme as demais, corolarialmente. Como entender esse experimento que distingue a dedução teoremática como um passo criativo, que explica o aspecto heurístico das ciências formais?

A distinção entre dedução corolarial e teoremática surge, em Peirce, a partir da análise que ele faz sobre o procedimento de prova na geometria euclidiana (CP 4.233). Ele observou que alguns teoremas de Euclides demandavam apenas uma simples observação da figura geométrica, ao passo que a maioria exigia um experimento sobre os diagramas. Esse experimento "engenhoso" consiste em adicionar novos ele- mentos ao diagrama, como linhas auxiliares, sem as quais não seria possível obter a conclusão. Como no seguinte exemplo da proposição 16, citada anteriormente.

Dada a premissa "Seja um triângulo $\mathrm{ABC}$, e tendo sido prolongado um de seus lados, $\mathrm{BC}$, até $\mathrm{D}^{\prime}$, pretende-se provar que " $\mathrm{O}$ ângulo exterior, sob ACD, é maior do que cada um dos ângulos sob CBA e BAC, interiores e opostos". Ocorre que não é possível fazer essa demonstração a partir da simples observação do diagrama das premissas, o que seria uma dedução corolarial. O próximo passo da prova exige uma construção, que consiste na introdução de linhas auxiliares:

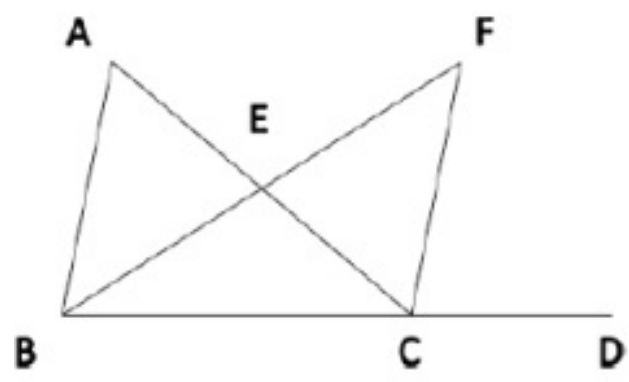

Imagem 3: Diagrama do passo construtivo da proposição 16 em Euclides (SHIN et al, 2018, p. 24).

Seciona-se o lado AC em dois em E (pela proposição I, 10) e, tendo sido ligada a $\mathrm{BE}$, prolonga-se sobre uma reta até o ponto F. Em seguida, seja EF igual a BE (I, 3), fique ligado por uma reta a FC.
Há um amplo debate entre comentadores do filósofo sobre o que exatamente consiste esse passo teoremático na prova dedutiva. Hintikka (1980), motivado pelos exemplos em geometria, in- 
terpretou a dedução teoremática como sendo a introdução de individuais auxiliares. Ketner (1985), entretanto, afirmou que a proposta de Peirce não pode ser restrita à introdução de novos individuais à prova, uma vez que sua concepção semiótica de diagramas é geral o bastante para incluir "não somente figuras, mas outras coisas, como álgebras, mapas, páginas em livros ou linguagem" (1985, p. 414).

Assim, um entendimento mais contemporâneo da dedução teoremática é mais próximo do que entendemos aqui por elemento criativo da prova. Dedução corolarial é aquela em que a demonstração é feita a partir da observação direta do diagrama das premissas, bastando para isso seguir axiomas e regras. É um procedimento mecânico que atesta o caráter explicativo do raciocínio dedutivo. Nenhuma criatividade está envolvida neste processo. Já na dedução teoremática não há clareza do próximo passo dedutivo a ser dado no processo inferencial, por isso fazse um movimento hipotético, um experimento que modifica os diagramas das premissas e que pode ser ou não eficaz para atingir o resultado ${ }^{13}$, Isso não significa, necessariamente, a introdução de novos individuais, mas uma escolha correta de quais regras usar de modo a seguir dedutivamente na demonstração do argumento (SHIN, 2016, p. 64-65; STJERNFELT, 2014, p. 291).

O ponto principal aqui é que esse passo teoremático envolve imaginação e criatividade para descobrir essas novas relações representadas diagramaticamente (EP 1, 228). A diagramação do argumento e sua observação fazem parte de todo procedimento dedutivo. O que difere um procedimento de natureza teoremática é a manipulação e transformação desse modelo na imaginação. "Definições prévias de resultados conhecidos [...] não implicam ou sugerem, por si só, esta nova ideia", mas, "ao contrário, uma hipótese criativa e imaginativa é requerida para que a nova ideia seja trazida ao raciocínio" (CAMPOS, 2010, p. 131; cf. HOFFMAN, 2003 e 2010; PIETARINEN \& BELLUCCI, 2016). É claramente um elemento abdutivo dentro de um processo dedutivo' ${ }^{14}$, que, como um todo, envolve dedução pura (corolarial) e teoremática. Isso mostra que a prova matemática não é tão automática e mecânica

\footnotetext{
${ }^{13}$ Algo próximo ao que, inspirado em jogos de xadrez, Hintikka (1997) chamou de regras estratégicas, em oposição a regras definitórias.

${ }^{14}$ Shin (2016) sugere que esse passo abdutivo é dado em um metanível no qual questiona-se qual é a melhor estratégia, a ser avaliada posteriormente em sua eficácia.
} 
quanto a tradição axiomática da lógica supunha, e explica como a dedução pode ser informativa.

\section{3 - Grafos existenciais}

Nossa segunda tese é a de que o raciocínio dedutivo teoremático, que explicita como a dedução é criativa, pode ser melhor analisado por meio de um sistema de dedução natural híbrido ou heterodoxo, no qual signos icônicos tenham precedência, em sua expressividade, sobre os simbólicos. Peirce desenvolveu tais sistemas, que ele chamou de Grafos Existenciais ${ }^{15}$, O primeiro sistema, chamado Alfa, corresponde, apro- ximadamente, à lógica proposicional clássica.

Ele apresenta dois elementos básicos: a folha de asserção (sheet of assertion), o espaço em branco no qual uma letra proposicional é um grafo que simboliza uma sentença; e o corte (cut), linhas ao redor dos grafos que equivalem à negação. Dois grafos justapostos são lidos como uma conjunção. Com esses elementos sintáticos básicos (acrescidos de letras sentenciais), outros operadores são facilmente interpretados. Note os seguintes exemplos de grafos, seguidos, cada um, da notação simbólica correspondente:
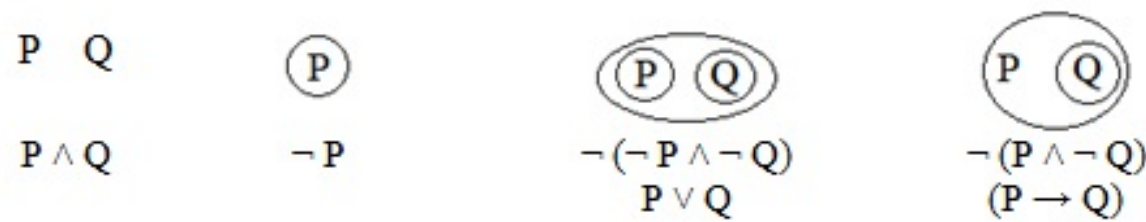

Imagem 4: Exemplos de grafos no sistema Alfa.

Em uma leitura simples, de fora para dentro e da esquerda para a direita, que Peirce chama endoporêutica ${ }^{16}$ (MS 650, pp. 18-19 apud. ROBERTS, 1973, p. 39, n. 13), o grafo que representa uma disjunção, por exemplo, é lido como "não é o caso que não-P e não-Q", e a implicação material como "não é o caso que $\mathrm{P}$ e não-Q". A interpretação desse sistema é, assim, mais intuitiva que sistemas puramente simbólicos.

Mas talvez uma maior inovação,

\footnotetext{
${ }^{15}$ Peirce nunca chegou a publicar suas descobertas, que têm sido objeto de investigação sobretudo entre seus scholars, como Zeman (1964), Roberts (1973), Shin (2002) e Sowa (2011).

${ }^{16}$ Para um método alternativo de leitura, cf. SHIN, 2002.
} 
antecipando os sistemas de dedução natural de Gentzen, seja o estabelecimento de regras de transformação para obtenção de provas. Consideremos a área positiva de um grafo como sendo aquela cercada por um número par de cortes ou nenhum corte, e área negativa, aquela cercada por um número ímpar de cortes. Teremos então regras de inserção e apagamentos de grafos e cortes, que podem ser formuladas em três pares simétricos:

\begin{tabular}{|l|l|}
\hline \multicolumn{1}{|c|}{ Regras de Inserção } & \multicolumn{1}{c|}{ Regras de Apagamento } \\
\hline $\begin{array}{l}\text { 1i: Qualquer grafo pode ser inserido } \\
\text { em uma área negativa. }\end{array}$ & $\begin{array}{l}\text { 1a: Qualquer grafo pode ser apagado } \\
\text { em uma área positiva. }\end{array}$ \\
\hline $\begin{array}{l}\text { 2i: Qualquer grafo pode ser copiado } \\
\text { na mesma área ou em outra cercada } \\
\text { por cortes adicionais. }\end{array}$ & $\begin{array}{l}\text { 2a: Qualquer grafo porventura ge- } \\
\text { rado por 2i pode ser apagado. }\end{array}$ \\
\hline $\begin{array}{l}\text { 3i: Um duplo corte pode ser inserido } \\
\text { em qualquer lugar. }\end{array}$ & $\begin{array}{l}\text { 3a: Um duplo corte pode ser apagado } \\
\text { em qualquer lugar. }\end{array}$ \\
\hline
\end{tabular}

Quadro 2: Regras de inferências do sistema Alfa.

Essas regras permitem provar teoremas e inferências do cálculo proposicional. Pela sua proximidade com os modernos sistemas de dedução natural, em nossos exemplos as regras serão aplicadas às premissas e as inferências serão justificadas no lado direito da página, conforme se costuma fazer em lógica simbólica. Comecemos com um exemplo simples, que representa o tipo de dedução que Peirce chamava corolarial, para provar um raciocínio em modus ponens. Em linguagem de grafos, o argumento é representado da seguinte forma:

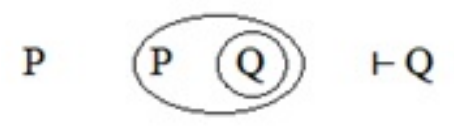

E a prova é a que segue:

1.

2.

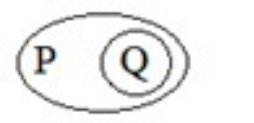

Premissas

$1,2 \mathrm{i}$ 


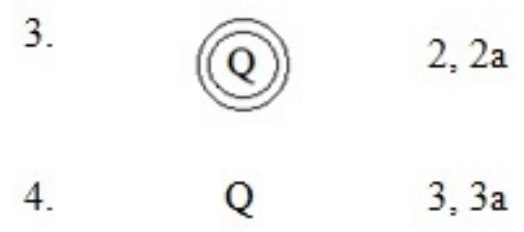

Essa prova, em quatro etapas, começa copiando a segunda premissa na folha de asserção pela regra (2i), para em seguida eliminar " $\mathrm{P}$ " pela regra de apagamento (2a). Por fim, aplica-se a eliminação de duplo corte (3a), de modo a obter a conclusão. Esse tipo de dedução não requer nenhuma estratégia, experimento ou manipulação de diagramas em sua demonstração. Pode-se observar a conclu- são representada no diagrama da segunda premissa, sendo explícita a relação de consequência lógica apresentada iconicamente na diagramação. Apenas são seguidas as regras, sem qualquer etapa que envolva criatividade imaginativa

Considere-se agora o seguinte exemplo: $(\mathrm{P} \rightarrow \mathrm{Q}),(\neg \mathrm{R} \rightarrow \neg \mathrm{Q})$ $\vdash(\mathrm{P} \rightarrow \mathrm{R})$. Em grafos, a representação seria da seguinte forma:
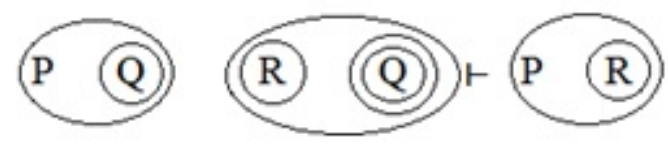

E demonstração desse argumento, a seguinte:

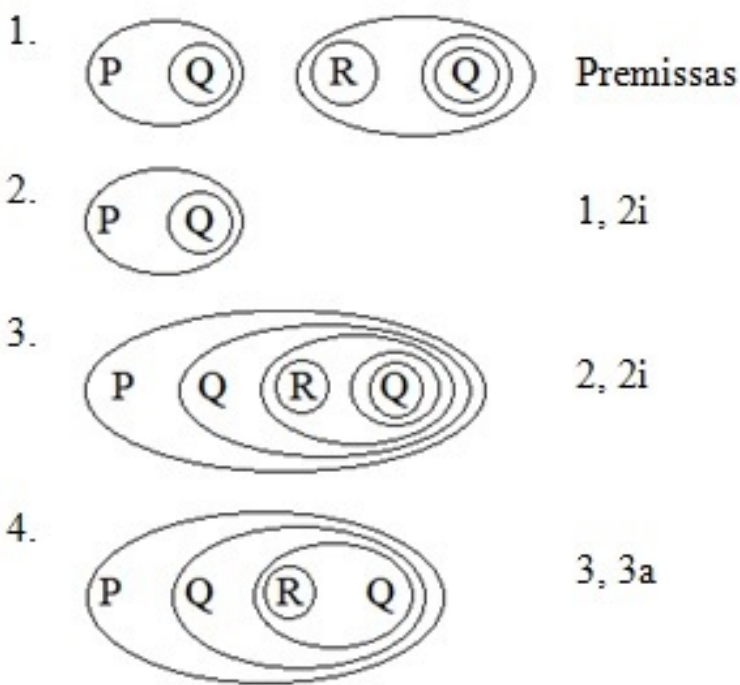


5.

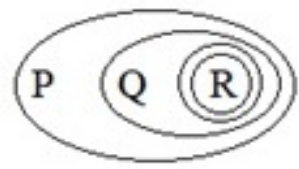

6.

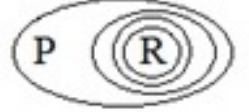

7.

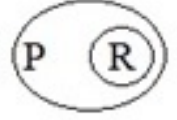

$4,2 a$

$5,1 \mathrm{a}$

$6,3 a$
Neste caso, observando-se as premissas não é possível visualizar a conclusão. Ela não é óbvia, trivial. O processo de demonstração, portanto, envolve uma experiência com a manipulação de uma das premissas, que é o terceiro passo da demonstração, quando, pela regra de inserção (2i), copiamos a segunda premissa dentro do escopo de $\neg$ Q, da primeira premissa.

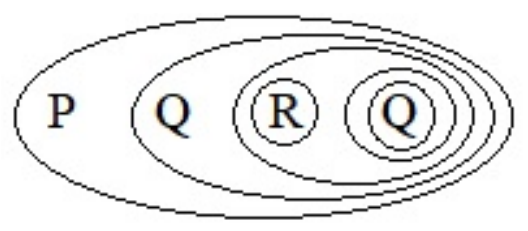

Esse é um passo que envolve um processo imaginativo e criativo (abdutivo), que pode ou não levar à conclusão que desejamos extrair. É o que Peirce chama de dedução teoremática. As demais etapas da prova seguem um método corolarial, com aplicação das regras de apagamento de duplo corte (3a), apagamento de grafo gerado por $2 \mathrm{i}(2 \mathrm{a})$, apagamento de grafo em área positiva (1a) e, finalmente, apagamento de duplo corte (3a).

Já sistema de grafos Beta pode ser compreendido como uma extensão do Alfa. A principal diferença notacional é a introdução de uma linha, chamada "linha de identidade", que designa a existência de individuais. Esse dispositivo sintático permite representar expressões quantificadas da lógica de predicados. De modo geral, o vocabulário continua econômico: as letras maiúsculas representam predicações, mas não há, como na lógica simbólica, constantes ou variáveis. A leitura de quantificadores existencial e universal, nessa sintaxe de caráter icônico, é dada visualmente, por meio da leitura dos cortes. Podemos representar assim as proposições categóricas: 


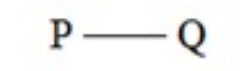

$\exists \mathrm{x}(\mathrm{Px} \wedge \mathrm{Qx})$

(i)

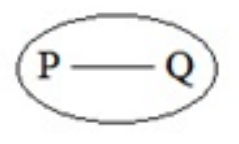

$\neg \exists \mathrm{x}(\mathrm{Px} \wedge \mathrm{Qx})$

(ii)

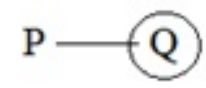

$\exists \mathrm{x}(\mathrm{Px} \wedge \neg \mathrm{Qx})$

(iii)

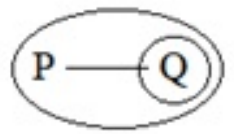

$\forall \mathrm{x}(\mathrm{Px} \rightarrow \mathrm{Qx})$

(iv)

Imagem 5: Exemplos de grafos no sistema Beta.

O primeiro diagrama acima (i) é lido como "existe um $\mathrm{P}$ que é $\mathrm{Q}$ " (particular afirmativa), enquanto o segundo (ii), adicionando um corte ao grafo, como "não é o caso que P e Q", ou seja, a "nenhum P é Q" (universal negativa). Inserindo o corte apenas em Q (iii), obtêmse “algum $P$ não é $Q$ ” (particular negativa), e o último grafo (iv) é interpretado como "não existe um

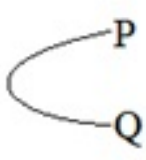

(v)

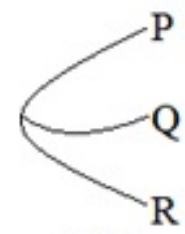

(vi)
P que não seja Q", ou "todo P é Q" (universal afirmativa). Uma linha de identidade não precisa ser reta, mas curva e com vários símbolos de predicado anexados às pontas ou ramos, chamados de ligaduras, conforme os grafos (v) e (vi) abaixo; uma ligadura sem um símbolo de predicado anexado é chamada de "ponta solta", como nos grafos (vii) e (viii):

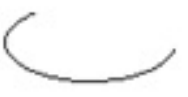

(vii)

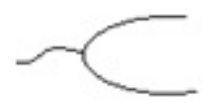

(viii)

Imagem 6: Exemplos de grafos no sistema Beta.

Para as regras de transformação são adicionadas, em comparação com as regras do sistema Alfa, ins- truções para manipular as linhas de identidade. 


\begin{tabular}{|c|c|}
\hline Regras de Inserção & Regras de Apagamento \\
\hline $\begin{array}{l}\text { 1i: Qualquer grafo pode ser inserido } \\
\text { e duas linhas de identidade podem } \\
\text { ser unidas em uma área negativa. }\end{array}$ & $\begin{array}{l}\text { 1e: Quaisquer grafos ou linhas de } \\
\text { identidade podem ser apagados em } \\
\text { uma área positiva. }\end{array}$ \\
\hline $\begin{array}{l}\text { 2i: Qualquer grafo pode ser copiado } \\
\text { na mesma área ou em outra cercada } \\
\text { por cortes adicionais, assim como: } \\
\text { a) pode ser adicionada qualquer li- } \\
\text { nha de identidade a um ramo com } \\
\text { uma "ponta solta" (desde que não re- } \\
\text { sulte em nenhum corte); } \\
\text { b) qualquer ponta solta de uma liga- } \\
\text { dura pode ser estendida para dentro, } \\
\text { através de cortes; } \\
\text { c) qualquer ligadura pode ser unida à } \\
\text { ligadura correspondente de um grafo } \\
\text { iterado. }\end{array}$ & $\begin{array}{l}\text { 2e: Qualquer grafo porventura gerado } \\
\text { por } 2 \mathbf{i} \text { pode ser apagado, assim como. } \\
\text { a) um ramo com uma ponta solta } \\
\text { pode ser retraído em qualquer linha } \\
\text { de identidade, desde que isso não re- } \\
\text { sulte em nenhum corte; } \\
\text { b) qualquer ponta solta de uma liga- } \\
\text { dura pode ser retraída para fora, atra- } \\
\text { vés de cortes. }\end{array}$ \\
\hline $\begin{array}{l}\text { 3i: Um duplo corte pode ser inse- } \\
\text { rido em qualquer lugar (e quaisquer } \\
\text { linhas que passem através dele po- } \\
\text { dem ser ignoradas). }\end{array}$ & $\begin{array}{l}\text { 3e: Um duplo corte pode ser apagado } \\
\text { em qualquer lugar (e quaisquer linhas } \\
\text { que passem através dele podem ser ig- } \\
\text { noradas). }\end{array}$ \\
\hline
\end{tabular}

Quadro 3: Regras de inferências do sistema Beta.

Essas definições teóricas são o suficiente para demonstramos o próximo caso de dedução teoremática. Esse é um dos exemplos de silogismos de Lewis Carroll (apud SUPES, 1957, p. 61), que adaptamos da seguinte forma:

Nenhum pato está disposto a valsar.

Nenhum funcionário público não está disposto a valsar.

Todas as minhas aves são patos.

Portanto, nenhuma das minhas aves são funcionários públicos.

Em linguagem de predicados, tem-se as seguintes fórmulas:

$$
\begin{gathered}
\forall \mathrm{x}(\mathrm{Px} \rightarrow \neg \mathrm{Qx}) \\
\forall \mathrm{x}(\mathrm{Rx} \rightarrow \mathrm{Qx}) \\
\forall \mathrm{x}(\mathrm{Sx} \rightarrow \mathrm{Px}) \\
\forall \mathrm{x}(\mathrm{Sx} \rightarrow \neg \mathrm{Rx})
\end{gathered}
$$


E, diagramado em GE, tem-se:
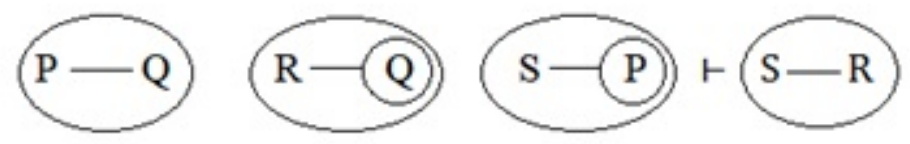

Observando-se as premissas, não é possível visualizar a conclusão do argumento, o que significa que não há um algoritmo que determina que regras usar para extraí-la. Portanto, é necessário um experimento, que é um passo hipotético-abdutivo na dedução. Como fazer esse experimento? Nota-se que a terceira premissa contém o termo " $\mathrm{S}$ ", fechado em ímpar, e a segunda, o termo " $R$ ", fechado também em ímpar. Assim, após diagramar as premissas, o passo (3) é um experimento, que consiste em diagramar a segunda premissa no grafo "P" da terceira premissa, fechado em par. Isso, contudo, não será o suficiente para extrair a conclusão. É preciso ainda um segundo experimento (4), no qual diagramamos a primeira premissa dentro do grafo da segunda. Esses passos são uma estratégia que pode ou não ser eficaz na demonstração do argumento.

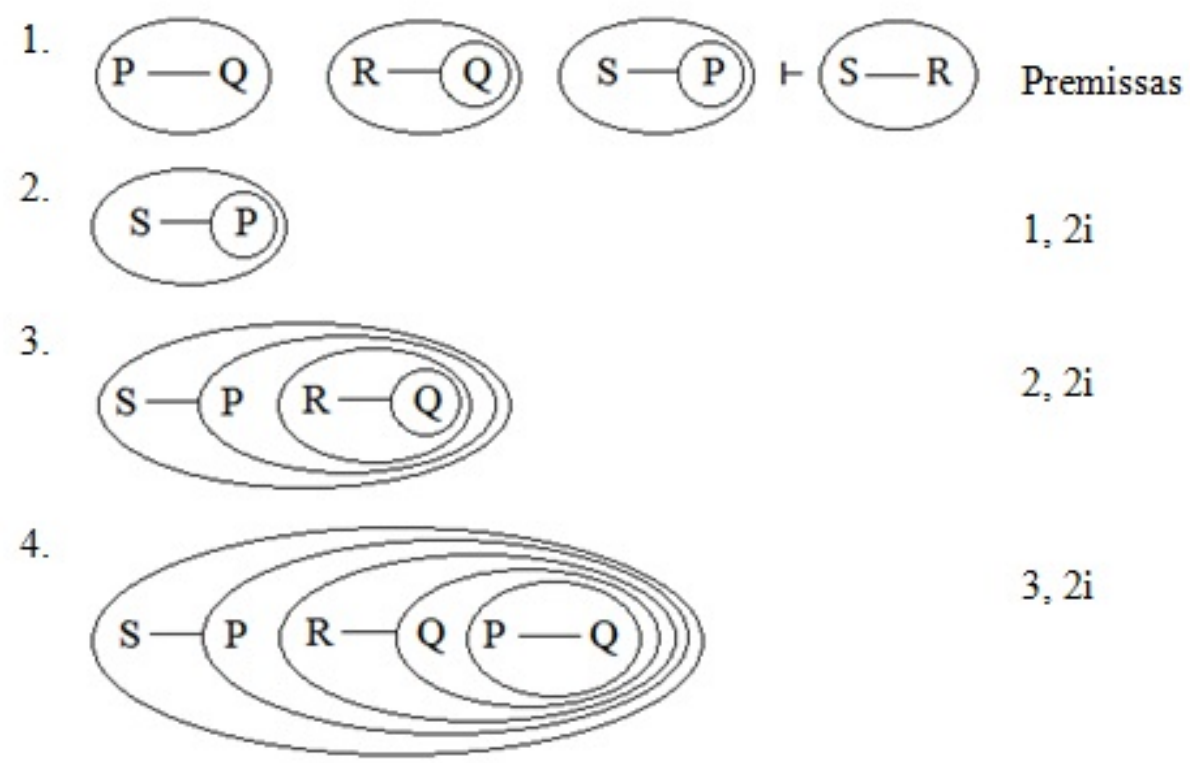

A essa dedução teoremá- etapas: primeiro, pelas regras de tica segue-se corolarialmente, inserção, une-se cada um dos símaplicando-se as regras em duas bolos de predicado por linhas de 
identidade; na segunda, pelas regras de apagamento, elimina-se os símbolos de predicado dos grafos inseridos pelas primeiras regras, assim como duplos cortes, chegando-se à conclusão (a prova inteira encontra-se em anexo).

\section{Conclusão}

Dedução entendida como consequência lógica, cuja prova é obtida por método mecânicos de aplicação de axiomas e regras, não explica como a lógica pode ser surpreendente e revelar aspectos criativos em seus procedimentos menos básicos. Neste artigo vimos como o conceito de dedução teoremática de Peirce ajuda a entender melhor a criatividade envolvida na lógica matemática. Dois aspectos fundamentais da dedução devem ser assim destacados: a iconicidade do objeto da lógica, o diagrama, cujas propriedades visuais são matéria de observação, e a manipulação ou experimentação que é feita com diagramas em uma etapa abdutiva e estratégica no processo inferencial dedutivo.

Por fim, mostramos como a dedução teoremática pode ser empiricamente testada, de modo mais eficiente, em um sistema de notação icônica como os Grafos Existenciais, elaborado por Peirce. Expusemos ainda dois exemplos nos quais a conclusão da dedução não poderia ser obtida a menos que fossem realizados experimentos hipotéticos e imaginativos sobre suas premissas. Esta etapa da prova, argumentamos, é um passo criativo dentro da dedução. Acreditamos que há desdobramentos dessas ideias instigantes de Peirce a serem exploradas em procedimentos heurísticos da lógica moderna.

\section{Referências}

ANDERSON, Douglas R. Creativity and the philosophy of C. S. Peirce. Martinus Nijhoff Publishers: Dordrecht, 1987.

CAMPOS, Daniel G. The imagination and hyphotesis-making in mathematics: a peircean account. In: New essays on Peirce's Mathematical Philosophy. MOORE, Matthew E. (ed.). Chicago and La Salle, Illinois: Open Court, 2010. p. 123-145.

EUCLIDES. Os elementos. Trad. e introd. Irineu Bicudo. São Paulo: UNESP, 2009.

FREGE, Gottlob (1879). Preface. In: Begriffsschrift, a formula language, modeled upon that of arithmetic, for pure thought. In: HEIJENOORT, Jean van. From Frege to Gödel: a source book in Mathematical Logic, 1879-1931. Cambridge, Mass.; London, England: Harvard University Press, 1967. p. 5-8.

HINTIKKA, Jaakko. Information, deduction, and the a priori. In: Logic, language-games and information. Kantian themes in the Philosophy of Logic. Oxford: Clarendon Press, 1973. p. 222-241. 
C.S. Peirce "first real discovery" and its contemporary relevance. In: The relevance of Charles Peirce. FREEMAN, Eugene (ed.). La Salle, Illinois: The Hegeler Institute/ Monist Library of Philosophy, 1983. p. 107-118.

. On creativity in reasoning. In: ANDERSSON, Ake E. and SAHLIN, Nils-Eric (eds.). The complexity of creativity.Dordrecht: Kluwer Academic Publishers, 1997. p. 67-78.

HOFFMAN, Michael. Peirce's "diagrammatic reasoning" as a solution of the learning paradox. In: Process pragmatism: essays on a quiet philosophical revolution. DEBROCK, Guy (ed.). Amsterdam/ New York: Rodopi B.V., 2003. p. 121-143.

. "Theoric transformations" and a new classification of abductive inferences. Transactions of the Charles S. Peirce Society: a quarterly journal in American Philosophy, v. 46, n. 4, p. 570-590, 2010.

KETNER, Kenneth Laine. How Hintikka misunderstood Peirce's account of theorematic reasoning. Transactions of the Charles Peirce Society: a quarterly journal in American Philosophy, vol. XXI, n. 3, p. 407-418, 1985.

LEVY, Stephen H. Peirce's theoremic/ corollarial distinction and the interconnections between mathematics and logic. In: Studies in the logic of Charles Sanders Peirce. HOUSER, Nathan; ROBERTS, Don D.; and EVRA, James Van (eds.). Indianapolis: Indiana University Press, 1997, p. 85-110.

LISZKA, James Jakób. A general introduction to the semeiotic of Charles Sanders Peirce. Bloomington and Indianapolis: Indiana University Press, 1996.

PEIRCE, Charles Sanders. Collected Papers. 8 vols. HARTSHORNE, Charles; HEISS, Paul and BURKS, Arthur (eds.). Cambridge: Harvard University Press, 1931-1958. [Citado como CP, seguido do número do volume e do número do parágrafo.]

. Essential Peirce. HOUSER, Nathan; KLOESEL, Christian (eds.). Vol. 1 (1867-1893). Bloomington: Indiana University Press, 1992. [Citado como EP 1, seguido do número da página.]

. Essential Peirce. The PEIRCE Edition Project (eds.). Vol. 2 (1893-1913). Bloomington: Indiana University Press, 1998. [Citado como EP 1, seguido do número da página.]

Pragmatism as a principle and method of right thinking: the 1903 Harvard lectures on pragmatism. TURRISI, Patricia Ann (ed.). New York: State University of New York Press, 1997. [Citado como HLP, seguido do número da página.]

. The new elements of mathematics by Charles S. Peirce. 4 vols. EISELE, Carolyn (ed.). Bloomington: Indiana University Press, 1976. [Citado como NEM seguido do volume e número da página.] Philosophy of Mathematics: selected writings. MOORE, Matthew E. (ed.). Indiana University Press: Bloomington and Indianapolis, 2010. [Citado como PM, seguido do número do parágrafo.]

PIETARINEN, Ahti-Veikko \& BELLUCCI, Francesco. New light on Peirce's conceptions of retroduction, deduction, and scientific reasoning. International Studies in the Philosophy of Science, v. 28, n. 4, p. 353-373, 2014.

. The iconic moment. towards a peircean theory of diagrammatic imagination. In: REDMOND, Juan, MARTINS, Olga P.; NEPOMUCENO, Ángel F. (eds). Epistemology, knowledge and the impact of interaction. Switzerland: Springer International Publishing, 2016. p 463-481.

RODRIGUES, Cassiano Terra. The method of scientific discovery in Peirce's Philosophy: deduction, induction, and abduction. Logica Universalis, v. 5, p. 127-164, 2011

SHIN, Sun-Joo. The iconic logic of Peirce's Graphs. Cambridge, Mass; London, England: The MIT Press, 2002.

. The mystery of deduction and diagrammatic aspects of representation. Review of Philosophy and Psychology, v. 6, n. 1, p. 49-67, 2015.

. The role of diagrams in abductive reasoning. In: KRAMER, Sybille and LJUNGBERG, Christina (Eds.). Thinking with Diagrams. Mouton: De Gruyter, 2016. p. 57-76.

. LEMON, Oliver and MUMMA, John, Diagrams. In: ZALTA, Edward N. (ed.). The Stanford Encyclopedia of Philosophy. Winter, 2018. Available in: <https://plato.stanford.edu/archives/win2018/entries/diagram

STJERNFELT, Frederik. Natural propositions: the actuality of Peirce's doctrine of dicisigns. Boston, Mass.: Docent Press, 2014.

SUPPES, Patrick. Introduction to logic. New York: Van Nostrand Reinhold Company, 1957.

ROBERTS, Don. The Existential Graphs of Charles S. Peirce. Mouton: The Hague, 1973.

SOWA, John F. Peirce's tutorial on existential graphs, Semiotica, n. 186, p. 345-394, 2011.

ZEMAN, J. Jay. The graphical logic of Charles S. Peirce. PhD. Dissertation. Department of Philosophy: University of Chicago, 1964. 


\section{ANEXO}

1.
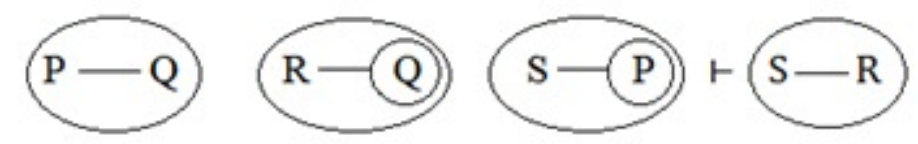

Premissas

2.

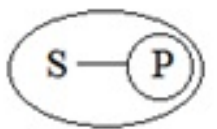

$1,2 \mathrm{i}$

3.

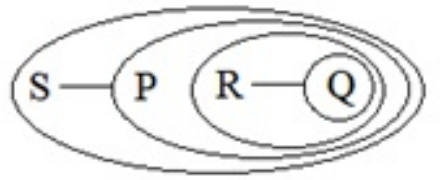

$2,2 \mathrm{i}$

4.

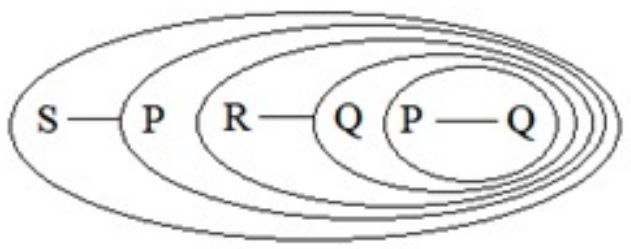

$3,2 \mathrm{i}$

5.

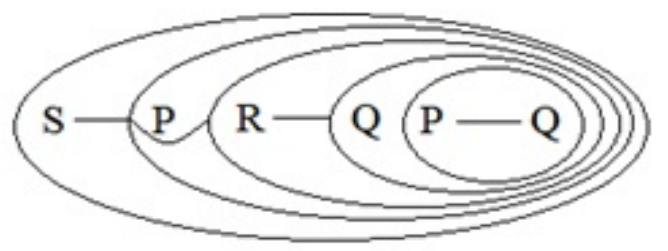

4, 2ia

6.

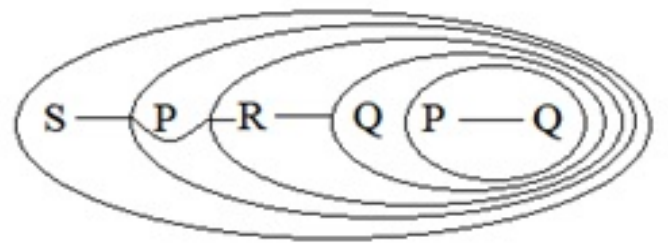

5, 2ib

7.

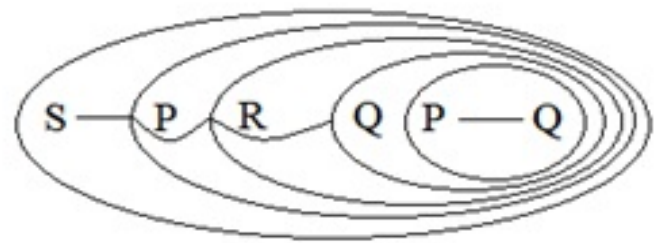

6, 2ic

8.

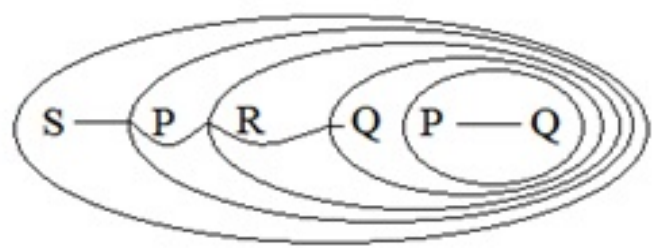

7, 2ib

9.

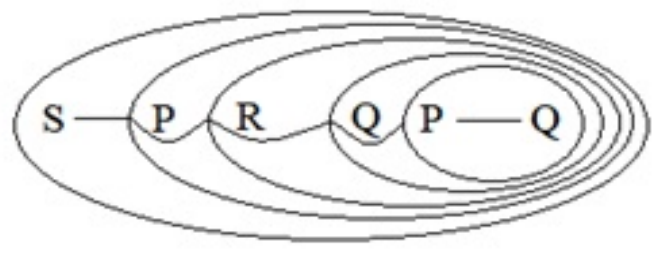

8,2 ic 
10

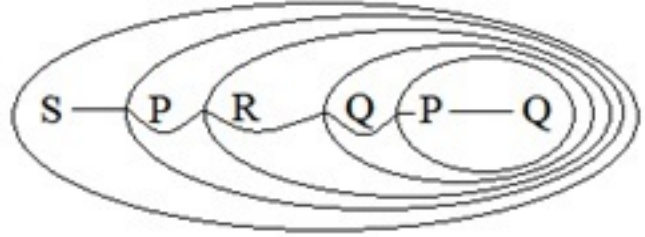

11.

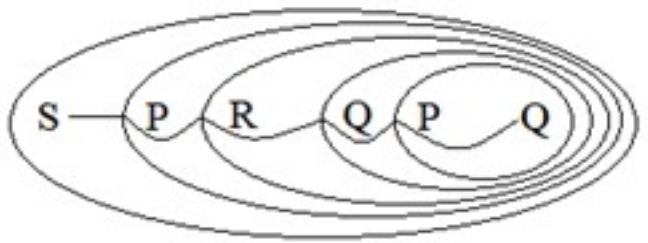

12.

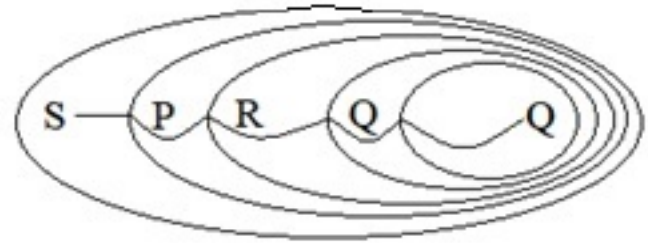

13.

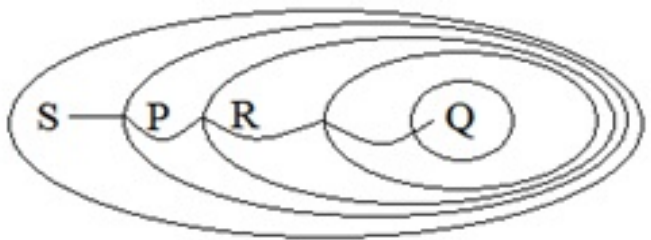

14.

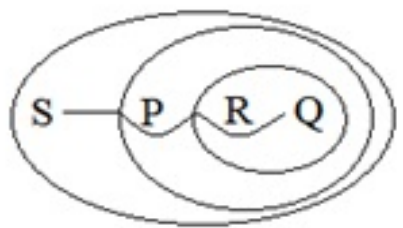

15.

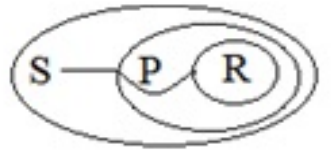

16.

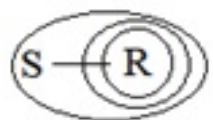

17.

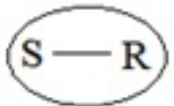

$9,2 \mathrm{ib}$

$10,2 \mathrm{ia}$

$11,2 \mathrm{e}$

$12,1 \mathrm{e}$

$13,3 \mathrm{e}$

$14,2 \mathrm{e}$

$15,1 \mathrm{e}$

$16,3 \mathrm{e}$

Recebido: 04/12/2019

Aprovado: 14/01/2020

Publicado: 26/01/2020 
